\title{
Kinase inhibitors fail to induce mesenchymal-epithelial transition in fibroblasts from fibrotic lung tissue
}

\author{
XUEFENG XU ${ }^{1,2}$, XUAN WAN $^{1,2}$, JING GENG $^{1,2}$, FEI LI $^{1,2}$, CHEN WANG $^{2,3}$ and HUAPING DAI ${ }^{1,2}$ \\ ${ }^{1}$ Department of Respiratory and Critical Care Medicine, Beijing Chao-Yang Hospital, \\ Capital Medical University; ${ }^{2}$ Beijing Key Laboratory of Respiratory and Pulmonary Circulation Disorders, \\ Beijing Institute of Respiratory Medicine, Beijing 100020; ${ }^{3}$ Beijing Hospital, Beijing 100730, P.R. China
}

Received February 20, 2013; Accepted April 23, 2013

DOI: $10.3892 / \mathrm{ijmm} .2013 .1415$

\begin{abstract}
Epithelial-mesenchymal transition (EMT) has been considered to be involved in idiopathic pulmonary fibrosis (IPF). However, the EMT process in vivo is much more complex and controversial. Studies regarding the opposite process, mesenchymal-epithelial transition (MET) in fibroblasts, are limited. Therefore, the aim of this study was to verify the involvement of the transforming growth factor (TGF)- $\beta 1$-dependent EMT network in the process of pulmonary fibrosis and to explore the possibility of MET. Fibrotic lung tissues were obtained from patients with IPF with histological evidence of usual interstitial pneumonia at the time of surgical lung biopsy. For the controls, histologically normal lung tissues were obtained from patients with primary spontaneous pneumothorax at the time of thoracoscopy with stapling of any air leaks. Real-time RT-PCR and western blot analysis revealed that the mRNA and protein levels of TGF- $\beta 1$, TGF- $\beta 1$ receptor type I/II/III (T $\beta R I / I I / I I I)$, Smad2/3/4 and Snail1/2 were significantly upregulated in the fibrotic lung tissue. Inhibitors of various kinases implicated in EMT, including TGF- $\beta 1 / \mathrm{Smad}$, Rho kinase (ROCK), p38 mitogen-activated protein kinase (p38 MAPK) and c-Jun NH-terminal kinase (JNK) were used to determine the MET potential in fibroblasts from fibrotic lung tissue. Western blot analysis or indirect immunofluorescence staining revealed that Smad inhibitor, as well as other kinase inhibitors failed to induce the MET process, determined by cellular morphology and protein markers. Our data suggest that the MET process may not be the exact reversal of EMT. In addition to using kinase inhibitors, other intervention measures should be used to explore the possibility of the MET process in fibroblasts from fibrotic lung tissue.
\end{abstract}

Correspondence to: Dr Huaping Dai, Department of Respiratory and Critical Care Medicine, Beijing Chao-Yang Hospital, Capital Medical University, 8th Gong Ren Ti Yu Chang South Road, Beijing 100020, P.R. China

E-mail: daihuaping@sina.com

Key words: epithelial-mesenchymal transition, idiopathic pulmonary fibrosis, mesenchymal-epithelial transition, Smad, Snai, transforming growth factor- $\beta 1$

\section{Introduction}

Idiopathic pulmonary fibrosis (IPF) is a chronic, progressive, lethal disease of unknown etiology and pathogenesis. It continues to be associated with considerable morbidity and mortality (1). The recent paradigm suggests that IPF is driven by chronic epithelial micro-injury and a subsequent deregulated repair process, leading to abnormal mesenchymal cell activation and proliferation and excess extracellular matrix (ECM) accumulation $(1,2)$. A determinant role in ECM deposition is played by myofibroblasts (3), which are $\alpha$-smooth muscle actin ( $\alpha$-SMA)-expressing fibroblasts and the main source of type I/III collagen, fibronectin and fibrogenic cytokines in fibrotic foci. However, the origin of fibroblasts in these fibrotic foci has not yet been fully elucidated. It was once believed that the migration, proliferation and activation of resident mesenchymal cells are the main sources of fibroblasts; however, emerging evidence has indicated that myofibroblasts may also be derived through the process of epithelial-mesenchymal transition (EMT) (4-7). Through this process, epithelial cells lose certain characteristics, such as apical-basolateral polarization, specialized cell-cell junctional structures and epithelial markers, and undergo cytoskeletal reorganization, ultimately acquiring the morphological and functional features of mesenchymal-like cells (8-10). The abnormal activation of EMT programs has been associated with an abnormal wound healing process and tissue fibrosis, cancer invasion and metastasis (8-10).

EMT is an orchestra which is delicately modulated by several signaling molecules, including transforming growth factor- $\beta$ (TGF- $\beta$ ), epidermal growth factor (EGF), hepatocyte growth factor (HGF), fiborblast growth factor (FGF) and integrin-linked kinase (ILK) (7). Among these, TGF- $\beta 1$ is a multifunctional cytokine that regulates cell proliferation, migration, or differentiation and is the master switch of EMT (11). TGF- $\beta 1$ is directly or indirectly recognized by 3 heterogenic cell surface receptors (types I, II and III) (12). Both the type I TGF- $\beta 1$ receptor (T $\beta \mathrm{RI})$ and type II receptor (T $\beta$ RII) are transmembrane serine/threonine kinases. Ligand binding to T $\beta$ RII and T $\beta$ RI induces the recruitment and phosphorylation of receptor-Smads (R-Smads, Smad2 and Smad3). Activated Smad2/3 heterodimerize with the Co-Smad (Smad4) to form a transcriptionally active complex which translocates 
to the nucleus and modulates the expression of TGF- $\beta$ target genes $(12,13)$.

EMT has been extensively investigated in vitro and data have shown that many different types of cells undergo EMT-like changes in response to TGF- $\beta 1$ stimulation $(14,15)$. The transition process of lung fibroblasts in vitro is mainly characterized by the downregulation or complete loss of epithelial markers, such as E-cadherin, cytokeratin, aquaporin 5 and prosurfactant protein (pro-SP) B, and the acquisition of new mesenchymal proteins, including vimentin, $\alpha$-SMA, fibroblast specific protein 1 (FSP1), type I collagen and fibronectin $(14,15)$. However, EMT-associated tissue fibrosis in vivo is a more complex and controversial phenomenon (16) and studies on the reverse process of EMT, mesenchymal-epithelial transition (MET), are limited. In this study, we hypothesized that the EMT program is activated in fibrotic lung tissue. Part of the injured alveolar epithelial cells undergo complete EMT-like changes and phenotypically resemble fibroblasts. Primarily cultured fibroblasts from fibrotic lung tissue are partly from epithelial cells. Kinase inhibitors targeting the EMT process may induce a possible MET process in these epithelial cellderived fibroblasts. In detail, we examined the expression level of the TGF- $\beta 1$ signaling network and found that TGF- $\beta 1$, T $\beta$ RI/II/III, Smad2/3/4 and Snail1/2 were significantly upregulated in the fibrotic lung tissue. We then examined the possibility of MET in cultured fibroblasts from fibrotic lung tissue using specific inhibitors of T $\beta R I$, Rho kinase (ROCK), p38 mitogen-activated protein kinase (p38 MAPK) and c-Jun NH-terminal kinase (JNK), and found that blocking TGF- $\beta 1$ and other kinase signals failed to induce the MET process.

\section{Materials and methods}

Subjects and lung tissue procurement. Lung tissue was obtained from 5 IPF patients (5 males; mean age, 58.6 \pm 4.7 years) with histological evidence of usual interstitial pneumonia at the time of surgical lung biopsy. The diagnosis of IPF was derived according to the standards accepted by the American Thoracic Society/European Respiratory Society (17). For the controls, histologically normal lung tissue was obtained from 3 patients (3 males; mean age, $20.8 \pm 2.8$ years) with primary spontaneous pneumothorax at the time of thoracoscopy with stapling of any air leaks.

This study was approved by the Ethics Committee of Beijing Chaoyang Hospital of Capital Medical University, Beijing, China and written informed consent was obtained according to institutional guidelines from all investigated subjects.

Cells and reagents. The explant culture method was used for the culture of primary fibroblasts. In brief, lung specimens were minced with scissors into pieces and washed with phosphate-buffered saline (PBS). Subsequently, 5-10 pieces were transferred into culture flasks (Corning Inc., Corning, NY, USA) with high glucose Dulbecco's modified Eagle's medium (DMEM), containing 10\% fetal bovine serum (FBS), penicillin $100 \mathrm{U} / \mathrm{ml}$ and streptomycin $100 \mathrm{mg} / \mathrm{ml}$. Tissues were then incubated in a humidified $5 \% \mathrm{CO}_{2}$ incubator at $37^{\circ} \mathrm{C}$. The medium were changed every 5 to 6 days. Approximately 3-4 weeks later, a monolayer of fibroblast-like cells fully covered the bottom of the flask. Th explant tissue was then removed and the cells were trypsinized and resuspended in supplemented DMEM for subculture. All reagents were purchased from HyClone (Logan, UT, USA). Cells at passages 4-8 (3 cell lines) were used for all the experiments. The identification and purity of the cultured primary lung fibroblasts were confirmed by morphological observation as well as immunostaining with vimentin, fibronectin and collagen I/III. The chemical inhibitors, SB203580, SP600125, Y27632 and SB431542 (Biotrend, Cologne, Germany) were aliquoted after reconstitution and frozen at $-20^{\circ} \mathrm{C}$.

Antibodies. The following antibodies were used in immunofluorescence and western blot analyses: mesenchymal cell markers included rabbit monoclonal anti- $\alpha$-SMA, rabbit monoclonal anti-vimentin, mouse polyclonal anti-collagen III, rabbit polyclonal anti-collagen I, rabbit polyclonal anti-fibronectin antibodies; epithelial cell marker, rabbit polyclonal antiE-cadherin antibodies; TGF- $\beta 1$ system antibodies included mouse monoclonal anti-TGF- $\beta 1$ (active form), rabbit monoclonal anti-T $\beta$ RI/II, mouse monoclonal anti-T $\beta$ RIII, rabbit monoclonal anti-(phospho)Smad2/3, rabbit monoclonal antiSnail and rabbit monoclonal anti-phospho AKT antibodies. All of the antibodies were obtained from Abcam (Cambridge, MA, USA) and were used according to the manufacturer recommendations.

$R N A$ purification and real-time RT-PCR. Total RNA was isolated from the fibrotic and normal lung tissue using the RNeasy MiniPrep kit (Tiangen Biotech, Beijing, China) and $2 \mu \mathrm{g}$ of RNA from each sample was reverse-transcribed using the Omniscript RT kit (Tiangen Biotech) using oligo(dT) primers $(1 \mu \mathrm{M})$ at $37^{\circ} \mathrm{C}$ for $1 \mathrm{~h}$. Real-time RT-PCR was performed on an ABI PRISM 7500 instrument (Applied Biosystems, Foster, CA, USA) using SYBR-Green PCR reagents (Tiangen Biotech). Reaction mixtures were incubated for $2 \mathrm{~min}$ at $94^{\circ} \mathrm{C}$ followed by 40 cycles of $15 \mathrm{sec}$ at $94^{\circ} \mathrm{C}$, $20 \mathrm{sec}$ at $55^{\circ} \mathrm{C}$ and $35 \mathrm{sec}$ at $68^{\circ} \mathrm{C}$. For each sample, gene expression was corrected against the $\beta$-actin mRNA level and the comparative threshold cycle number $(\mathrm{Ct})$ method was used to assess the relative quantification of gene expression. The fold-change of the target genes were calculated using the $2^{-\triangle \Delta C T}$ method. The primers used for real-time PCR are shown in Table I.

Protein extraction, SDS-PAGE and indirect immunoblot analysis. Cells were harvested and lysed in radioimmunoprecipitation assay (RIPA; Pierce, Rockford, IL, USA) buffer supplemented with complete proteinase and phosphatase inhibitor cocktails (Roche, Basel, Switzerland). Protein extracts were clarified by centrifugation $(12,000 \mathrm{rpm}, 15 \mathrm{~min})$ at $4^{\circ} \mathrm{C}$ and the concentrations were determined using the bicinchoninic acid assay kit (Pierce). Equal amounts $(30 \mu \mathrm{g})$ of protein extracts were then separated by $8-12 \%$ sodium dodecyl sulfate-polyacrylamide gel electrophoresis (SDS-PAGE) and transferred onto polyvinylidene difluoride (PVDF) membranes (Millipore, Billerica, MA, USA). The membranes were then blocked with $5 \%$ non-fat dry milk or bovine serum albumin (BSA) in TBS (10 mM Tris-HCl, pH 7.6; $150 \mathrm{mM} \mathrm{NaCl} ; 0.1 \%$ Tween-20) and incubated with the indicated primary antibodies for overnight 
Table I. Primers used for real-time RT-PCR.

\begin{tabular}{lll}
\hline Gene & \multicolumn{1}{c}{ Forward sequences } & \multicolumn{1}{c}{ Reverse sequences } \\
\hline Smad4 & 5'-TGATGTCTGAGAAGATGTCC-3' & 5'-GCATCGACAGAGACATACAG-3' \\
TGF- $\beta 1$ & 5'-GAGCCTGAGGCCGACTACTA-3' & 5'-CGGAGCTCTGATGTGTTGAA-3' \\
T $\beta$ RII & 5'-CGGAGCTCTGATGTGTTGAA-3' & 5'-AGCAACTGCAGCATCACCTC-3' \\
Snail1 & 5'-CAACAGTAACAATAGGGCAG-3' & 5'-GAGCTGCAGGACTCTAATCCA-3' \\
Snail2 & 5'-CGGTGGGGTTGAGGATCT-3' & 5'-TGGTTGCTTCAAGGACACAT-3' \\
Smad2 & 5'-GTTGCAGTGAGGGCAAGAA-3' & 5'-ATCCTAACAGAACTTCCGCC-3' \\
$\beta$-actin & 5'-TGCTATCCAGGCTGTGCTAT-3' & 5'-AGTCCATCACGATGCCAGT-3'
\end{tabular}

at $4^{\circ} \mathrm{C}$ according to the manufacturer's instructions. After washing, membranes were incubated for $1 \mathrm{~h}$ with appropriate secondary, HRP-labeled antibodies (Proteintech, Chicago, IL, USA). Finally, an enhanced chemiluminescence detection (ECL) buffer (KPL, Kirkegaard \& Perry Laboratories, Inc., Gaithersburg, MD, USA) with ChemiDoc XRS (Bio-Rad, Hercules, CA, USA) was used for the visualization of the protein bands. Relative protein levels were determined by densitometry using Quantity One Software (Bio-Rad) or ImageJ software and normalized by mouse anti- $\beta$-actin monoclonal antibody (mAb) or mouse anti-GAPDH mAb (both from Abcam, Cambridge, CA, USA) when the detected protein has a similar molecule weight with $\beta$-actin.

Indirect immunofluorescence staining. Cells were seeded on glass coverslips and cultured as described above. The slides were then rinsed once with ice-cold PBS and fixed with $4 \%$ paraformaldehyde for $20 \mathrm{~min}$ at room temperature. The cells were then permeabilized in PBS containing 0.1\% Triton X-100. After rinsing in PBS and blocking with 5\% BSA at room temperature for $1 \mathrm{~h}$, monolayers were incubated with the appropriate primary antibody overnight at $4^{\circ} \mathrm{C}$. After extensive washing and blocking with 5\% BSA at room temperature for $30 \mathrm{~min}$, the slides were incubated with appropriate secondary antibodies conjugated to fluorescein isothiocyanate (FITC) or Texas Red (KPL) at room temperature in a humidified chamber in the dark. The coverslips were then overturned on a microscope slide containing one drop of anti-fade solution with DAPI. Images were then taken at room temperature using an Olympus inverted microscope (Olympus, Tokyo, Japan).

Statistical analysis. Unless otherwise indicated, all experiments were performed on 3 separate occasions, each time with triplicates. For statistical evaluation, the results are presented as the means \pm SD. For comparisons between groups, we used the Mann-Whitney or Kruskal-Wallis test with SPSS software. A P-value $<0.05$ was considered to indicate a statistically significant difference.

\section{Results}

TGF- $\beta 1$-dependent EMT-related $m R N A$ expression is increased in fibrotic lung tissues of patients with IPF. We wished to investigate whether TGF- $\beta 1$-dependent EMT-related mRNA expression was upregulated in lungs from patients with IPF.
A

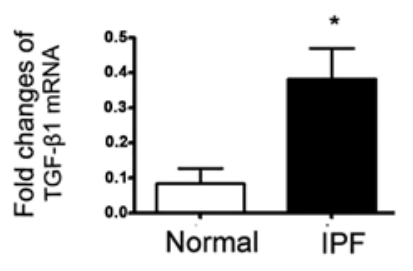

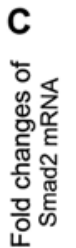

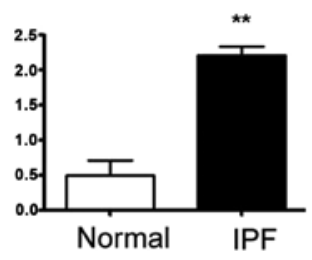

E

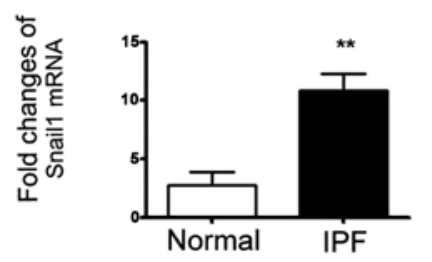

Figure 1. TGF- 31 -dependent epithelial-mesenchymal transition (EMT)-related mRNA expression was increased in fibrotic lung tissue. Lung tissue lysates were prepared and relative (A) TGF- $\beta 1$, (B) T $\beta R I I$, (C) Smad2, (D) Smad4, (E) Snail1 and (F) Snail2 RNA levels were determined by real-time RT-PCR. ${ }^{*} \mathrm{P}<0.05$, ${ }^{* *} \mathrm{P}<0.01$. Idiopathic pulmonary fibrosis (IPF), $\mathrm{n}=5$; control, $\mathrm{n}=3$.

IPF and control lung tissues were lysed in RL buffer and whole RNA was extracted according to the manufacturer's instructions. Real-time RT-PCR was then performed to determine the mRNA expression levels of TGF- $\beta 1, T \beta R I I, \operatorname{Smad} 2, \mathrm{Smad} 4$, Snail1 and Snail2. We found that compared to the normal lung tissue, the mRNA expression of TGF- $\beta 1$, T $\beta$ RII, Smad2, Smad4, Snail1 and Snail2 was significantly increased in the fibrotic human lungs. These results indicated that the mRNA levels are overexpressed during EMT in fibrotic tissue (Fig. 1).

TGF- $\beta 1$-dependent EMT-related protein expression is upregulated in fibrotic lung tissues of patients with IPF. We also performed western blot analysis for the detection of the levels of proteins involved in the TGF- $\beta 1$-dependent EMT program, 

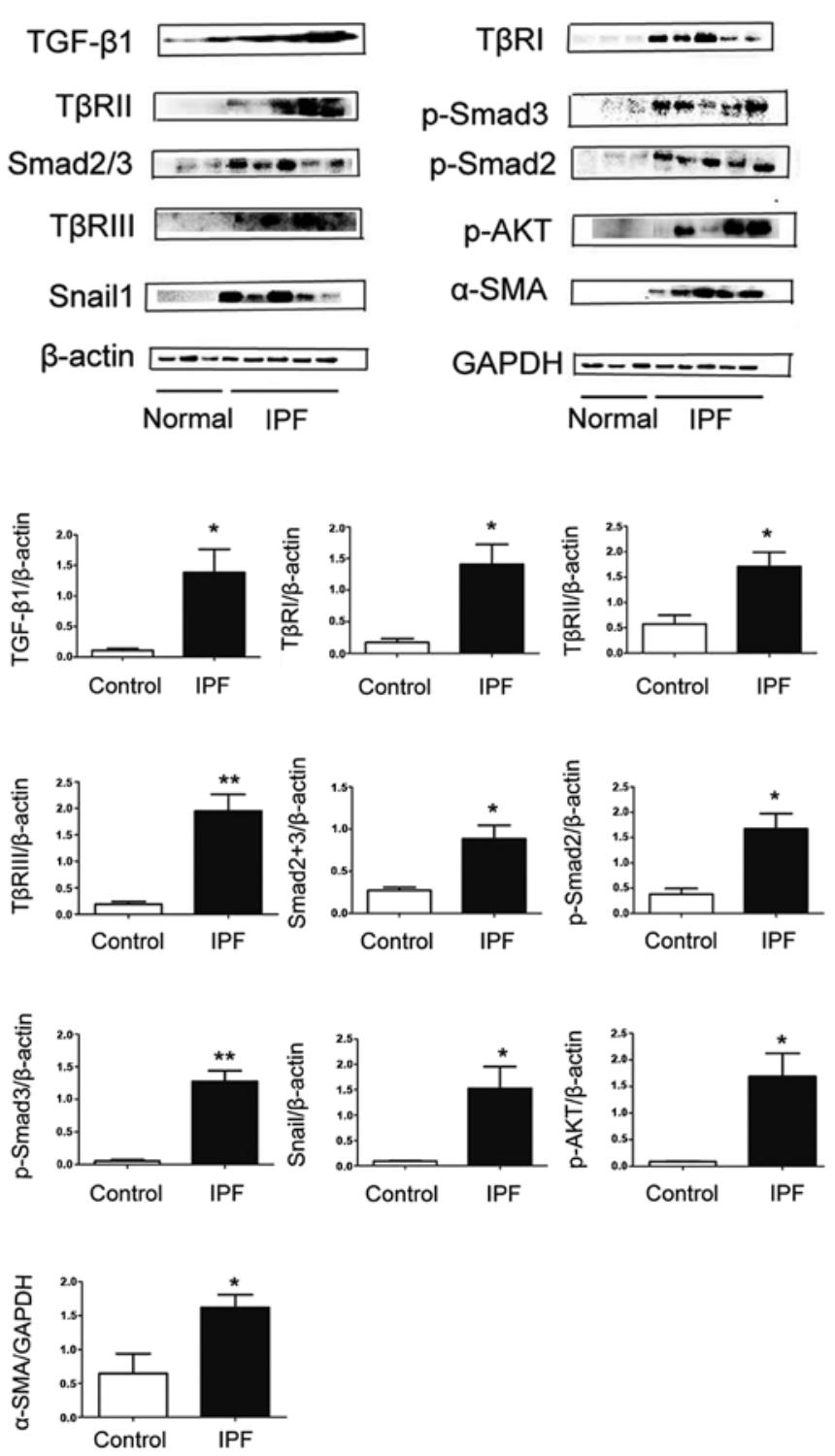

Figure 2. Representative results of (A) western blot analysis for epithelialmesenchymal transition (EMT)-related proteins. (B) Tissue lysates were prepared and relative TGF- $\beta 1, T \beta R I, T \beta R I I, T \beta R I I I, S m a d 2 / 3, p-S m a d 2$, p-Smad3, Snail, p-AKT and $\alpha$-SMA protein levels were determined by western blot analysis and normalized to $\beta$-actin or GAPDH (when the targeted protein has a similar molecular weight with $\beta$-actin, we used GAPDH instead of the internal control gene). Data were presented as the means $\pm \mathrm{SE}$ ${ }^{*} \mathrm{P}<0.05,{ }^{* *} \mathrm{P}<0.01$. Idiopathic pulmonary fibrosis (IPF), $\mathrm{n}=5$; control, $\mathrm{n}=3$.

such as TGF- $\beta 1$, T $\beta$ RI, T $\beta$ RII, T $\beta$ RIII, Smad $2 / 3$, p-Smad2, p-Smad3, Snail, p-AKT and $\alpha$-SMA. Tissue lysates were obtained by RIPA buffer, and primary antibodies were probed to detect the expression of EMT-related proteins. Fig. 2A shows the representative results of western blot analysis. Compared with the normal control tissue, in the lung tissue obtained from patients with IPF, the levels of TGF- $\beta 1$-dependent EMT-related proteins, such as TGF- $\beta 1, T \beta R I, T \beta R I I, T \beta R I I I$, Smad2/3, p-Smad2, p-Smad3, Snail, p-AKT and $\alpha$-SMA were significantly increased (Fig. 2B).

Characterization of human lung fibroblast cultures. In order to explore the potential MET process, cultures of primary fibroblasts from fibrotic lung tissue were established from sterile peripheral lung tissue biopsies. After 3-4 weeks, fibroblasts 'crawled' from the explants and proliferated to form a single layer of adherent cells (Fig. 3B). The cells were then trypsinized and passaged. At up to 8 serial passages, all cells displayed typical spindle-shaped morphology under a phasecontrast light microscope (Fig. 3A) and stained positive for vimentin, collagen I (COLI), collagen III (COLIII), fibronectin (FN), TGF- $\beta 1$, T $\beta R I, T \beta R I I, T \beta R I I I$, Snail and Smad2/3 (Fig. 4). All subsequent experiments were performed using subconfluent quiescent cultures of human lung fibroblasts between passages 4 and 8 to maintain comparability. These data showed that human lung fibroblasts were successfully cultured and that TGF- 31 -dependent EMT markers were expressed by these cells.

TRRI inhibitor, SB431542 does not induce EMT reversal. We hypothesized that part of these cultured fibroblasts from fibrotic lung tissue were derived from abnormal alveolar epithelial cells and would undergo MET changes by possible intervention. We first wished to examine the effect of the kinase inhibitor, SB431542, targeting TGF- $\beta 1 / T \beta R I$ activity, since TGF- $\beta 1$ is the master switch of EMT $(7,14,15)$. Subsequently, quiescent cells were incubated with SB431542 at $5 \mu \mathrm{M}$ or the vehicle for 24, 48, 72 and $96 \mathrm{~h}$. As shown in Fig. 5A, the addition of SB431542 at $5 \mu \mathrm{M}$ for $96 \mathrm{~h}$ was insufficient to induce an elongated morphological change of lung fibroblasts to the cubical pattern. Furthermore, as shown by our results, SB431542 failed to reduce the protein level of vimentin, a mesenchymal marker, as indicated by indirect immunofluorescence and western blot analysis (Fig. 5B and C). These data demonstrated that inhibiting the TGF- $\beta 1$ signal was insufficient to promote the MET process.

A combination of kinase inhibitors targeting T $\beta R I, R O C K, p 38$ MAPK and JNK does not induce the MET process. We then aimed to determine whether a combination of different kinase inhibitors can induce the MET potential of human lung fibroblasts. We wished to determine the effects of 4 different kinase inhibitors specifically targeting T $\beta$ RI, ROCK, p38 MAPK and JNK (SB431542, Y27632, SB203580 and SP60012, respectively), as these kinases have previously been proven to play a specific role in the EMT process $(15,18-21)$. Firstly, to examine whether the inhibition of the T $\beta R I$ and ROCK pathways (using SB431542 and Y27632, respectively) can induce a significant MET process, we performed the following experiments: quiescent subconfluent cells were treated with SB431542 and Y27632 at $5 \mu \mathrm{M}$ for $24,48,72$ and $96 \mathrm{~h}$. The induction of the MET potential was evaluated by morphological observation, indirect immunofluorescence and western blot analysis for vimentin and E-cadherin expression. Although the fibroblasts became even more elongated following treatment with the inhibitors, they still did not assume an epithelial phenotype (Fig. 6A). Furthermore, the epithelial marker (E-cadherin) and mesenchymal marker (vimentin) were not affected (Fig. 6B and C). Taken together, these data show that the MET process of lung fibroblasts may not be induced by the inhibition of T $\beta R I$ and ROCK.

We then used inhibitors targeting T $\beta$ RI, ROCK, p38 MAPK and JNK (SB431542, Y27632, SB203580 and SP60012, respec- 

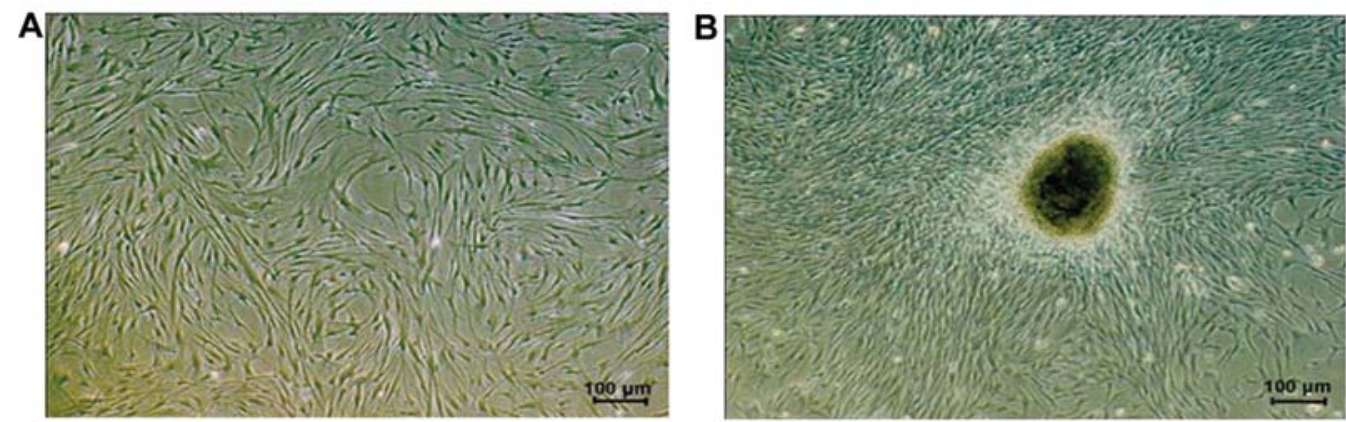

Figure 3. Phase-contract light microscopy characterization of fibroblasts from fibrotic lung tissue. (A) Phase-contrast microscopy observation of fibroblasts from fibrotic lung tissue. Cells displayed typical spindle-shaped morphology (bar shows $100 \mu \mathrm{m}$ ). (B) Cell cultures of fibroblasts from fibrotic lung tissue were grown out from sterile peripheral lung tissue biopsies and cultured in DMEM medium containing 10\% fetal bovine serum (FBS), penicillin 100 U/ml, streptomycin $100 \mathrm{mg} / \mathrm{ml}$. After 3-4 weeks, a monolayer of fibroblast like cells fully covered the bottom of the flask (bar shows $100 \mu \mathrm{m}$ ).
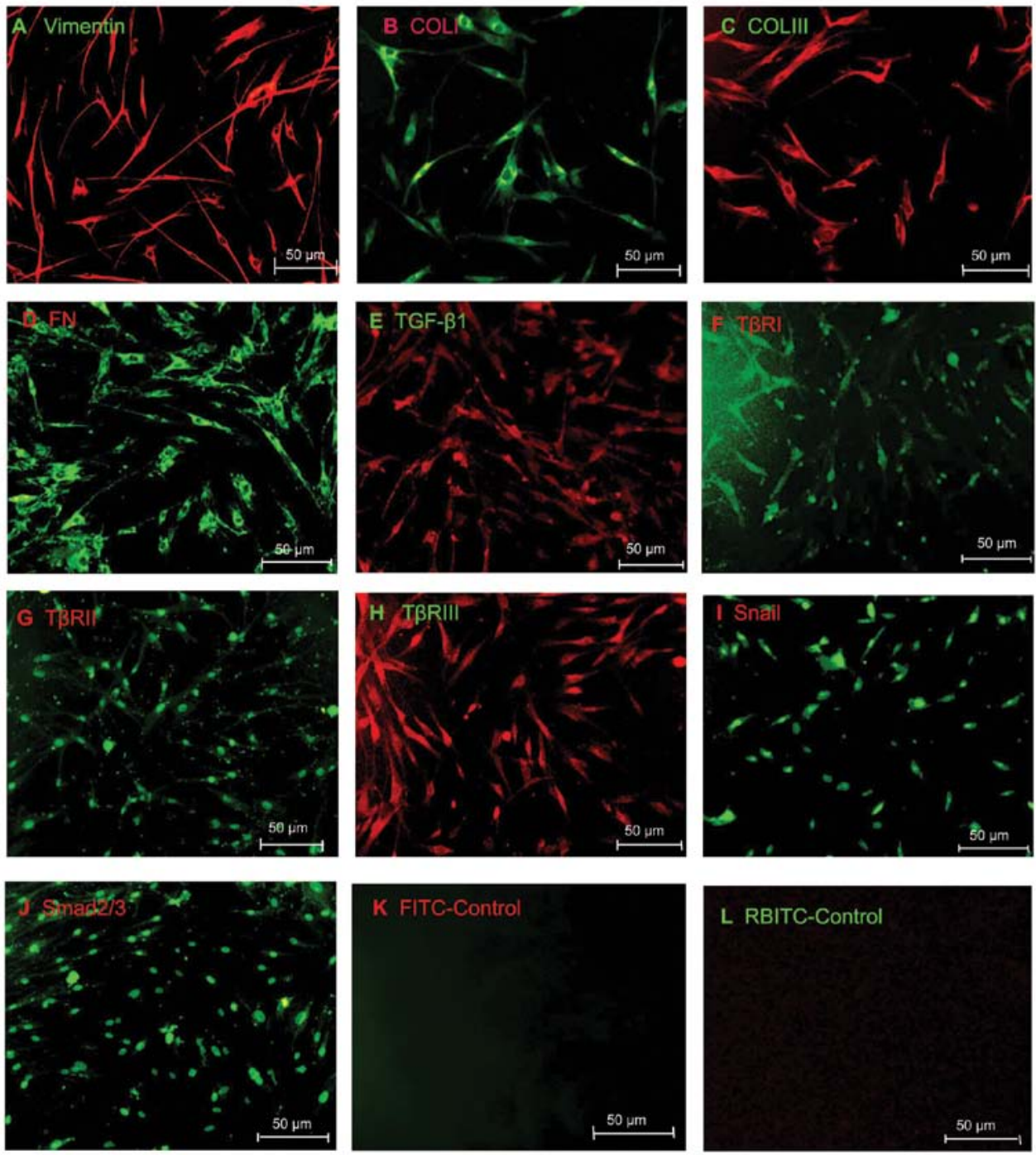

Figure 4. Indirect immunofluorescence assay of fibroblasts from fibrotic lung tissue. The phenotype and epithelial-mesenchymal transition (EMT) markers of the fibroblasts from fibrotic lung tissue was determined by indirect immunofluorescence assay. Fibroblasts were grown on the slides and fixed with $4 \%$ paraformaldehyde. Non-specific protein binding was blocked by incubating the slides in PBS containing 4\% bovine serum albumin (BSA) for $1 \mathrm{~h}$. The slides were then incubated overnight at $4^{\circ} \mathrm{C}$ with primary antibodies specific for (A) vimentin, (B) COLI, (C) COLIII, (D) FN, (E) TGF- $\beta 1$, (F) T $\beta R I$, (G) T $\beta R I I$, (H) T $\beta R I I I$, (I) Snail and (J) Smad2/3. After washing, the slides were further incubated for 1 min with either FITC- or RBITC-coupled anti-rabbit IgG or anti-mouse IgG. After washing, the preparations were mounted with FluorSave reagent and observed under a microscope. (K and $\mathrm{L}$ ) show negative control. (Bar shows $50 \mu \mathrm{m}$ ).

tively), to determine whether they can induce synergistic effects on the induction of MET. The cells were treated with $5 \mu \mathrm{M}$
$\mathrm{SB} 431542,5 \mu \mathrm{M}$ Y27632, $1 \mu \mathrm{M} \mathrm{SB} 203580$ and $10 \mu \mathrm{M} \mathrm{SP} 60012$ for $24,48,72$ and $96 \mathrm{~h}$. Phase-contrast morphology showed 


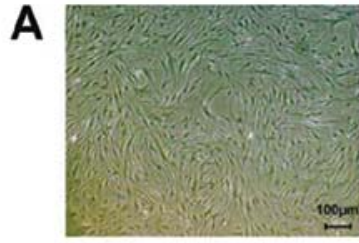

Control

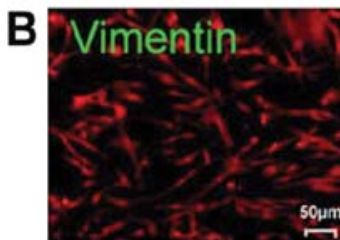

Control

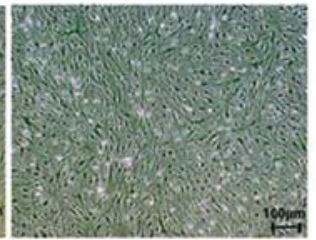

SB431542 $(5 \mu \mathrm{M})$

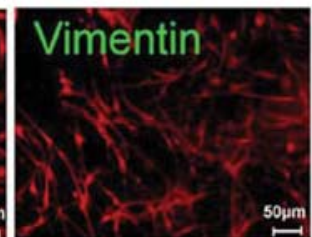

SB431542 $(5 \mu \mathrm{M})$

\section{C}

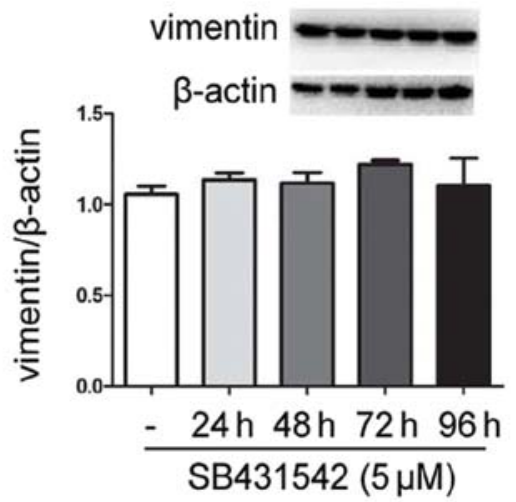

Figure 5. Treatment of fibroblasts from fibrotic lung tissue with SB431542 failed to induce the mesenchymal-epithelial transition (MET) process. Human fibroblasts from fibrotic lung tissue were incubated with $5 \mu \mathrm{M}$ SB431542, a T $\beta R I$ inhibitor, for 24, 48, 72 and $96 \mathrm{~h}$. (A) Phase-contract microscopy observation was performed to examine the morphological changes of fibroblasts after treatment with SB431542 for $96 \mathrm{~h}$ (bar shows $100 \mu \mathrm{m}$ ). (B) A representative example of indirect immunofluorescence assay. Cells were grown on slides and fixed with $4 \%$ paraformaldehyde. After washing and blocking with PBS (containing $4 \%$ BSA), the slides were incubated with primary antibodies specific for vimentin. After washing, the slides were further incubated for 1 min with RBITC-coupled anti-mouse IgG. After washing, the slides were observed under a microscope (bar shows $50 \mu \mathrm{m}$ ). (C) Western blot analysis showing the expression level of vimentin in fibroblasts. Values represent the means \pm SE of 3 independent experiments.

A

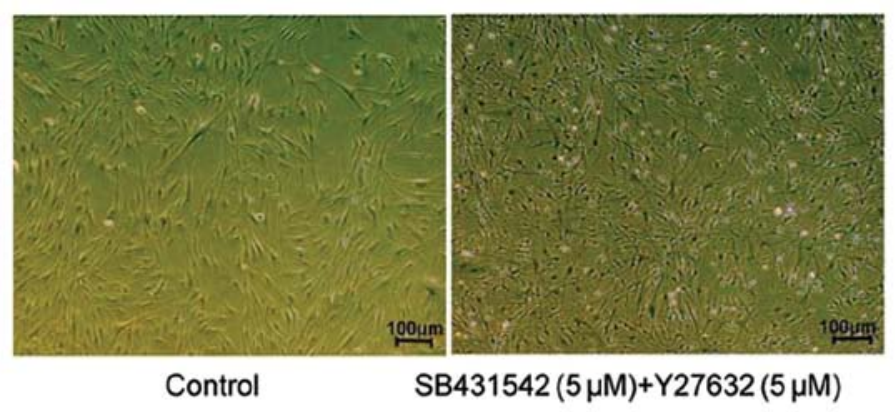

B

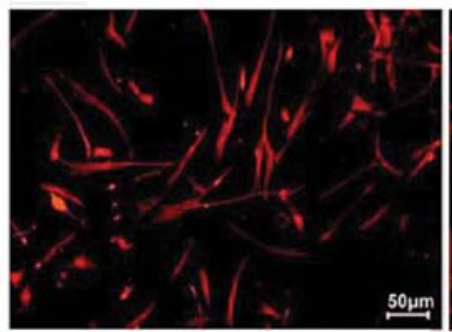

Control

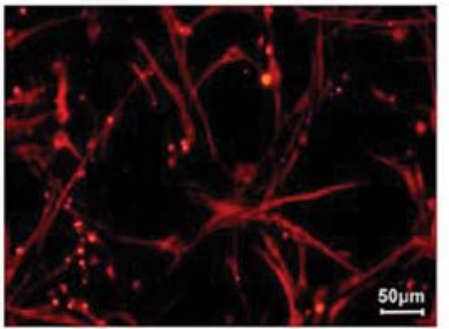

SB431542 $(5 \mu M)+Y 27632(5 \mu M)$
C

\section{vimentin \\ $\beta$-actin}

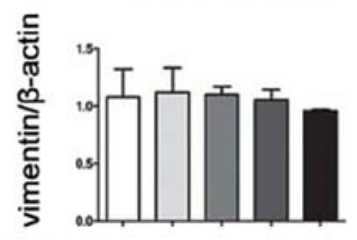

SB431542 $(5 \mu \mathrm{M})-++++$

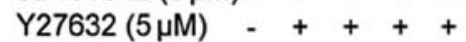

Time (h) $\quad-24 \quad 487296$

\section{E-cadherin}

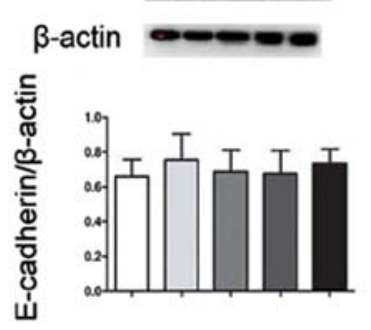

SB431542 $(5 \mu \mathrm{M})-++++$

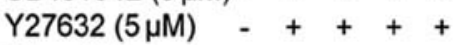

Time (h)

- 24487296

Figure 6. Combined treatment of fibroblasts from fibrotic lung tissue with T $\beta R I$ and RhoA inhibitors failed to induce the mesenchymal-epithelial transition (MET) process. Human fibroblasts from fibrotic lung tissue were incubated with SB431542 and Y27632 at $5 \mu \mathrm{M}$ for 24, 48, 72 and $96 \mathrm{~h}$. (A) Phase-contract microscopy observation was performed to examine the morphological changes of the fibroblasts after treatment with SB431542 and Y27632 for $96 \mathrm{~h}$ (bar shows $100 \mu \mathrm{m}$ ). (B) A representative example of indirect immunofluorescence assay showing vimentin expression (bar shows $50 \mu \mathrm{m}$ ). (C) Western blot analysis showing the expression level of vimentin in fibroblasts. Values represent the means \pm SE of 3 independent experiments. (D) Western blot analysis showing the expression level of E-cadherin in fibroblasts. Values represent the means \pm SE of 3 independent experiments.

no typical phenotypic changes (Fig. 7A). The expression of vimentin was not significantly affected by these inhibitors
(Fig. 7B and C). We then used these kinase inhibitors to treat fibroblasts for an even longer period of time (from 1 to 8 days). 

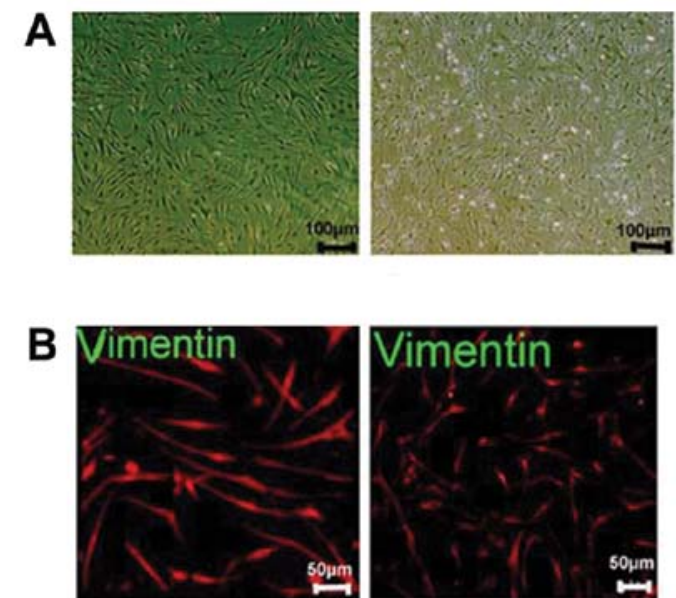

Control
SB431542 $(5 \mu \mathrm{M})$ Y27632 $(5 \mu \mathrm{M})$ SB203580 $(1 \mu \mathrm{M})$ SP600125 $(10 \mu \mathrm{M})$

\section{C}
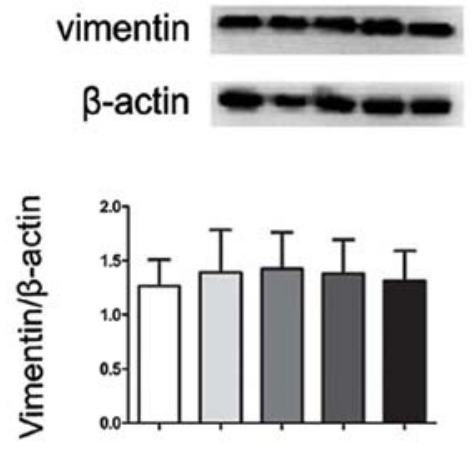

$\begin{array}{llllll}\text { SB431542 } & - & + & + & + & + \\ \text { Y27632 } & - & + & + & + & + \\ \text { SB203580 } & + & + & + & + \\ \text { SP600125 } & + & + & + & + & + \\ \text { Time (h) } & - & 24 & 48 & 72 & 96\end{array}$

Figure 7. Combined treatment of fibroblasts from fibrotic lung tissue with T $\beta$ RI, Rho A, p38 MAPK, JNK inhibitors failed to induce the mesenchymalepithelial transition (MET) process. Human fibroblasts from fibrotic lung tissue were incubated with $5 \mu \mathrm{M} \mathrm{SB} 431542,5 \mu \mathrm{M} \mathrm{Y} 27632,1 \mu \mathrm{M}$ SB203580 and $10 \mu \mathrm{M}$ SP60012 for 24, 48, 72 and $96 \mathrm{~h}$. (A) Phase-contract microscopy observation was performed to examine the morphological changes of fibroblasts after treatment with SB431542, Y27632, SB203580 and SP60012 for $96 \mathrm{~h}$ (bar shows $100 \mu \mathrm{m}$ ). (B) A representative example of indirect immunofluorescence assay showing vimentin expression (bar shows $50 \mu \mathrm{m}$ ). (C) Western blot analysis showing the expression level of vimentin in fibroblasts. Values represent the means \pm SE of 3 independent experiments.
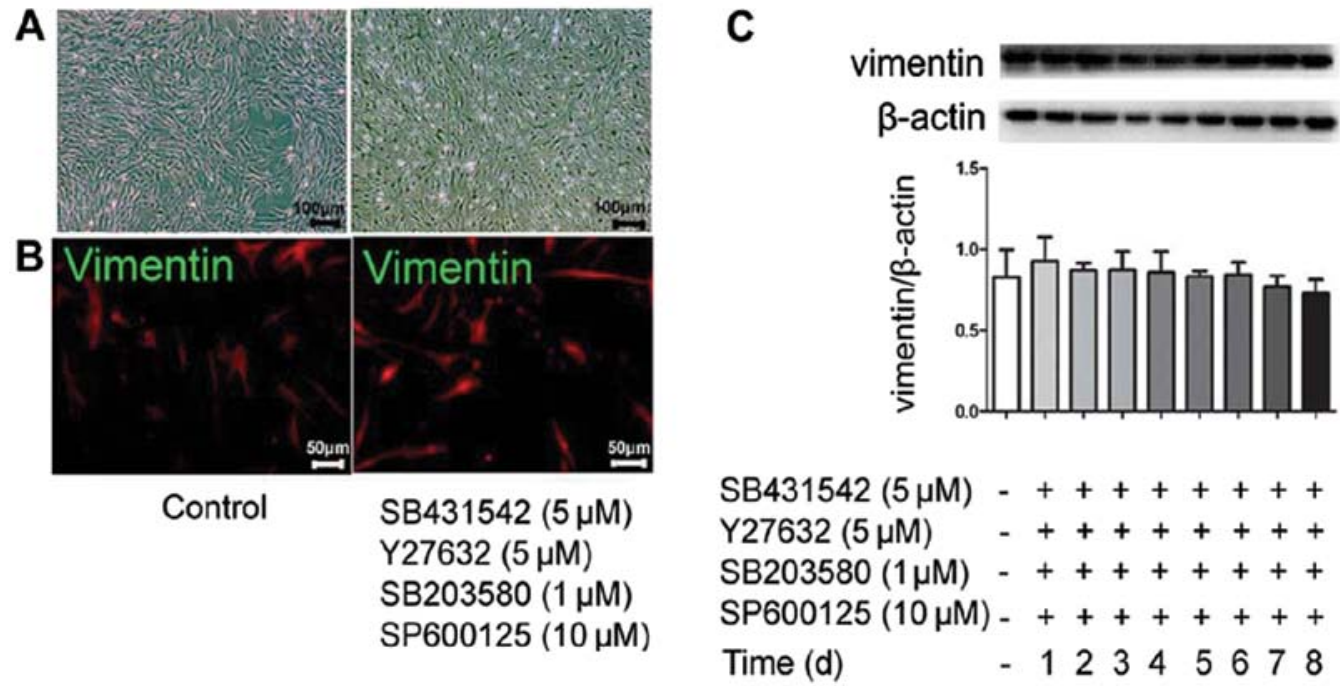

Figure 8. Longer combined treatment of human fibroblasts from fibrotic lung tissue with T $\beta R I$, Rho A, p38 MAPKand JNK inhibitors failed to induce the mesenchymal-epithelial transition (MET) process. Human fibroblasts from fibrotic lung tissue were incubated with $5 \mu \mathrm{M} \mathrm{SB} 431542,5 \mu \mathrm{M}$ Y27632, $1 \mu \mathrm{M}$ SB203580 and $10 \mu \mathrm{M}$ SP60012 for 1-8 days. (A) Phase-contract microscopy observation was performed to examine the morphological changes of fibroblasts after SB431542, Y27632, SB203580 and SP60012 treatment for 8 days (bar shows $100 \mu \mathrm{m}$ ). (B) A representative example of indirect immunofluorescence assay showing vimentin expression (bar shows $50 \mu \mathrm{m}$ ). (C) Western blot analysis showing the expression level of vimentin in fibroblasts. Values represent the means \pm SE of 3 independent experiments.

This effort also failed to induce the MET process (Fig. 8). Taken together, these results demonstrate that MET may be a delicate process that is not easily induced in fibroblasts.

\section{Discussion}

It is increasingly being recognized that injured epithelial cells can give rise to fibroblast-like cells and may thus contribute to the pathogenesis of fibrosis by undergoing EMT. In the present study, we hypothesized that part of cultured fibroblasts from fibrotic lung tissue are derived from abnormal epithelial cells and aimed to induce a possible MET process. We showed that the TGF- $\beta 1$-dependent EMT network (including TGF- $\beta 1$,

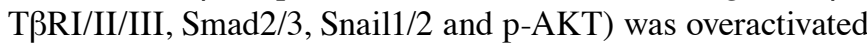
in the lung tissues of patients with IPF. We also successfully cultured primary human lung fibroblasts from lung explants. 
However, we failed to induce MET changes in cultured fibroblasts from fibrotic lung tissue by using kinase inhibitors targeting T $\beta$ RI, RhoA, p38 MAPK and JNK, all of which have been proven to contribute to the EMT process. Taken together, these data demonstrate that although the EMT program exists and is activated in IPF lung tissue, the direct induction of the MET process in fibroblasts from fibrotic lung tissue is a more daunting job to achieve.

EMT in vivo is a complex and controversial process, and its contribution to fibrotic disorders has not yet been elucidated. In this study, we used fibrotic lung tissue from patients IPF, or normal lung tissue from patients with primary spontaneous pneumothorax, to explore the activation of the TGF- $\beta 1$ dependent EMT network in fibrotic lung tissue. Western blot analysis and real-time RT-PCR revealed that TGF- $\beta 1$ signaling molecules involved in the EMT process, including TGF- $\beta 1$, T $\beta R I, T \beta R I I, T \beta R I I I$, Smad2, Smad3, Snail1 and Snail2 were upregulated and activated in fibrotic lung tissue. This indicated that EMT in vivo is possible and that epithelial cells may be one of the sources of mesenchymal cells. Several parallel studies are in line with our study. A previous study using gene array experiments demonstrated that genes which stimulate EMT, such as TGF- $\beta 3$, lymphoid enhancer factor-1 (LEF-1) and Slug (22), were upregulated in samples from patients with IPF. They suggested that the increased TGF- $\beta$ expression, decreased bone morphogenetic protein (BMP)-2 expression, and active BMP inhibition by gremlin created an EMT-favoring environment in IPF lungs (22). Another study, using immunohistochemical analysis, revealed that ATII cells assumed mesenchymal markers, such as N-cadherin, TGF- $\beta 1$ and collagen I, indicating a possible ongoing EMT process in epithelial cells (23). However, a previous study, using dual-immunohistochemistry assay, demonstrated that mesenchymal markers, such as $\alpha$-SMA and vimentin were not found in cells with epithelial markers in a bleomycin-induced pulmonary fibrosis mouse model and patients with IPF and non-specific interstitial pneumonia (24). These results suggest that EMT does not occur in IPF or bleomycin-induced pulmonary fibrosis in mice. Another possibility is that EMT may occur in pulmonary fibrosis but the detectable level of mesenchymal markers expressed in epithelial cells is too low to be detected by double immunohistochemistry (24).

Although previous studies have focused on the EMT process induced by TGF- $\beta 1$ or other profibrotic cytokines $(15,25-27)$, studies on the EMT reversal (MET) are limited. We hypothesized that if EMT in vivo really exists in IPF, then fibroblasts in fibrotic lung tissues may partly originate from injured epithelial cells. We also hypothesized that by blocking the activated signaling network in primarily cultured fibroblasts associated with EMT, at least some fibroblasts may abandon their mesenchymal markers and resume epithelial markers and the EMT reversal (MET) may become possible. To this end, we used kinase inhibitors targeting T $\beta$ RI, RhoA, p38 MAPK and the JNK pathways, all of which are involved in the EMT process. However, to our disappointment, the treatment of fibroblasts with the kinase inhibitors (either separately or together) failed to induce the MET process, indicated by morphology observation, indirect immunostaining and western blot analysis for epithelial and mesenchymal markers (E-cadherin and vimentin). These results indicate that the MET processs may not be the exact reversal of EMT; the process by which cells can alter their mesenchymal phenotype to resume an epithelial one is a much more complex one.

To date, studies on MET in fibrotic lung fibroblasts are limited. There are several studies, however, on MET in epithelial cells. Epithelial cells were fist incubated with TGF- $\beta 1$ or other cytokines to induce the EMT process, and subsequently, interference methods were used to block or reverse the EMT process (28-30). A previous study used murine renal tubular epithelial cells to explore the possibility of the MET process. They found that exposing cells to the T $\beta$ RI inhibitor, SB431542, combined with the ROCK inhibitor, Y27632, eliminated detectable actin stress fibers and mesenchymal gene expression while restoring epithelial E-cadherin and kidney-specific cadherin (Ksp-cadherin) expression (28). Another study used A549 and RLE-6TN (human and rat) alveolar epithelial-like cells, demonstrating that FGF-1 plus heparin reversed the morphological changes induced by TGF- $\beta 1$ and returned the epithelial and mesenchymal markers to the control levels (29). However, a previous study used primary human proximal tubule epithelial cells (RPTEC) and immortalized (HK-2) human proximal tubule epithelial cells to show that bone BMP-7 over a broad concentration range $(0.01-100 \mu \mathrm{g} / \mathrm{ml})$ failed to attenuate TGF- $\beta 1$-induced EMT in RPTEC or HK-2 cells (30). As discussed above, we also used fibroblasts from fibrotic lung tissue and incubated these cells with different inhibitors of kinases involved in EMT modulation to explore the potential of MET, but failed. Taken together, our data, as well as data from other studies illustrate that MET may be possible but should be induced carefully with different treatment methods.

In conclusion, although a limitation of the present study was the small sample size used to examine the activation of the TGF- $\beta 1$-induced EMT program, our data reveal that EMT in vivo is possible and contributes to the fibrotic process. Although our efforts to induce the MET process in human lung fibroblasts failed, future sutdies should focus on the MET process directly in abnormal fibroblasts from fibrotic tissue rather than in epithelial cells, as the MET process may not be the exact reversal of EMT.

\section{Acknowledgements}

This study was supported by grants from the National Natural Science Foundation of China (no. 30971312) and the Key Project of Beijing Municipal Education Commission Sci-Tech Development Program (no. KZ201110025028). We would like to thank Dr Bin You, Dr Jinbai Miao, Dr Qirui Chen, Dr Bo Tian and Dr Jin Zhang, Department of Thoracic Surgery, Beijing Chao-Yang Hospital for their kind supply of the lung tissue specimens from informed patients. We also thank Professor Jun Wang, Associate Researcher Yan Liang, Associate Researcher Xingyuan Jia, Research Assistant Ran Miao, Research Assistant Dong Leng, Research assistant Xiaoxi Huang, and Research Assistant Ying Wang, Beijing Key Laboratory of Respiratory and Pulmonary Circulation Disorders, for their kind technical assistance.

\section{References}

1. King TE Jr, Pardo A and Selman M: Idiopathic pulmonary fibrosis. Lancet 378: 1949-1961, 2011. 
2. Selman M, Thannickal VJ, Pardo A, Zisman DA, Martinez FJ and Lynch JP III: Idiopathic pulmonary fibrosis: pathogenesis and therapeutic approaches. Drugs 64: 405-430, 2004.

3. Phan SH: Biology of fibroblasts and myofibroblasts. Proc Am Thorac Soc 5: 334-337, 2008.

4. Lee $\mathrm{K}$ and Nelson CM: New insights into the regulation of epithelial-mesenchymal transition and tissue fibrosis. Int Rev Cell Mol Biol 294: 171-221, 2012.

5. Coward WR, Saini G and Jenkins G: The pathogenesis of idiopathic pulmonary fibrosis. Ther Adv Respir Dis 4: 367-388, 2010.

6. Gharaee-Kermani M, Hu B, Phan SH and Gyetko MR: Recent advances in molecular targets and treatment of idiopathic pulmonary fibrosis: focus on TGFbeta signaling and the myofibroblast. Curr Med Chem 16: 1400-1417, 2009.

7. Chapman HA: Epithelial-mesenchymal interactions in pulmonary fibrosis. Annu Rev Physiol 73: 413-435, 2011.

8. Kalluri R and Weinberg RA: The basics of epithelial-mesenchymal transition. J Clin Invest 119: 1420-1428, 2009.

9. Acloque H, Adams MS, Fishwick K, Bronner-Fraser M and Nieto MA: Epithelial-mesenchymal transitions: the importance of changing cell state in development and disease. J Clin Invest 119: 1438-1449, 2009.

10. Thiery JP, Acloque H, Huang RY and Nieto MA: Epithelialmesenchymal transitions in development and disease. Cell 139: 871-890, 2009.

11. Kalluri R and Neilson EG: Epithelial-mesenchymal transition and its implications for fibrosis. J Clin Invest 112: 1776-1784, 2003.

12. Lan HY and Chung AC: Transforming growth factor-beta and Smads. Contrib Nephrol 170: 75-82, 2011.

13. Pohlers D, Brenmoehl J, Loffler I, Müller CK, Leipner C, Schultze-Mosgau S, Stallmach A, Kinne RW and Wolf G: TGF-beta and fibrosis in different organs - molecular pathway imprints. Biochim Biophys Acta 1792: 746-756, 2009.

14. Willis BC, Liebler JM, Luby-Phelps K, Nicholson AG, Crandall ED, du Bois RM and Borok Z: Induction of epithelial-mesenchymal transition in alveolar epithelial cells by transforming growth factor-beta1: potential role in idiopathic pulmonary fibrosis. Am J Pathol 166: 1321-1332, 2005.

15. Kasai H, Allen JT, Mason RM, Kamimura T and Zhang Z: TGF-betal induces human alveolar epithelial to mesenchymal cell transition (EMT). Respir Res 6: 56, 2005.

16. Fragiadaki M and Mason RM: Epithelial-mesenchymal transition in renal fibrosis - evidence for and against. Int J Exp Pathol 92: 143-150, 2011.

17. American Thoracic Society; European Respiratory Society: American Thoracic Society/European Respiratory Society International Multidisciplinary Consensus Classification of the Idiopathic Interstitial Pneumonias. This joint statement of the American Thoracic Society (ATS), and the European Respiratory Society (ERS) was adopted by the ATS board of directors June 2001 and by the ERS Executive Committee, June 2001. Am J Respir Crit Care Med 165: 277-304, 2002.
18. Hutchison N, Hendry BM and Sharpe CC: Rho isoforms have distinct and specific functions in the process of epithelial to mesenchymal transition in renal proximal tubular cells. Cell Signal 21: 1522-1531, 2009.

19. Lv ZM, Wang Q, Wan Q, Lin JG, Hu MS, Liu YX and Wang R: The role of the p38 MAPK signaling pathway in high glucoseinduced epithelial-mesenchymal transition of cultured human renal tubular epithelial cells. PLoS One 6: e22806, 2011.

20. Kolosova I, Nethery D and Kern JA: Role of Smad2/3 and p38 MAP kinase in TGF- $\beta 1$-induced epithelial-mesenchymal transition of pulmonary epithelial cells. J Cell Physiol 226: 1248-1254, 2011.

21. van der Velden JL, Guala AS, Leggett SE, Sluimer J, Badura EC and Janssen-Heininger YM: Induction of a mesenchymal expression program in lung epithelial cells by wingless protein (Wnt)/ $\beta$-catenin requires the presence of $\mathrm{c}$-Jun $\mathrm{N}$-terminal kinase-1 (JNK1). Am J Respir Cell Mol Biol 47: 306-314, 2012.

22. Selman M, Pardo A and Kaminski N: Idiopathic pulmonary fibrosis: aberrant recapitulation of developmental programs? PLoS Med 5: e62, 2008

23. Lomas NJ, Watts KL, Akram KM, Forsyth NR and Spiteri MA Idiopathic pulmonary fibrosis: immunohistochemical analysis provides fresh insights into lung tissue remodelling with implications for novel prognostic markers. Int J Clin Exp Pathol 5: 58-71, 2012.

24. Yamada M, Kuwano K, Maeyama T, Hamada N, Yoshimi M, Nakanishi Y and Kasper M: Dual-immunohistochemistry provides little evidence for epithelial-mesenchymal transition in pulmonary fibrosis. Histochem Cell Biol 129: 453-462, 2008.

25. Zhang M, Zhang Z, Pan HY, Wang DX, Deng ZT and Ye XL: TGF-beta1 induces human bronchial epithelial cell-to-mesenchymal transition in vitro. Lung 187: 187-194, 2009.

26. Kim JH, Jang YS, Eom KS, Hwang YI, Kang HR, Jang SH, Kim CH, Park YB, Lee MG, Hyun IG, Jung KS and Kim DG: Transforming growth factor betal induces epithelial-to-mesenchymal transition of A549 cells. J Korean Med Sci 22: 898-904, 2007.

27. Fernando RI, Castillo MD, Litzinger M, Hamilton DH and Palena C: IL-8 signaling plays a critical role in the epithelialmesenchymal transition of human carcinoma cells. Cancer Res 71: 5296-5306, 2011.

28. Das S, Becker BN, Hoffmann FM and Mertz JE: Complete reversal of epithelial to mesenchymal transition requires inhibition of both ZEB expression and the Rho pathway. BMC Cell Biol 10: 94, 2009.

29. Ramos C, Becerril C, Montano M, Garcia-De-Alba C, Ramirez R, Checa M, Pardo A and Selman M: FGF-1 reverts epithelial-mesenchymal transition induced by TGF-\{beta\}1 through MAPK/ERK kinase pathway. Am J Physiol Lung Cell Mol Physiol 299: L222-L231, 2010.

30. Dudas PL, Argentieri RL and Farrell FX: BMP-7 fails to attenuate TGF-betal-induced epithelial-to-mesenchymal transition in human proximal tubule epithelial cells. Nephrol Dial Transplant 24: 1406-1416, 2009. 